\title{
New estimates for the number of integer polynomials with given discriminants
}

Natalia Budarina

Dundalk Institute of Technology

Vasilii Bernik

Institute of Mathematics, Minsk

Hugh O'Donnell

Technological University Dublin, hugh.odonnell@tudublin.ie

Follow this and additional works at: https://arrow.tudublin.ie/ittsciart

Part of the Mathematics Commons

\section{Recommended Citation}

Budarina, Natalia; Bernik, Vasilii; and O'Donnell, Hugh, "New estimates for the number of integer polynomials with given discriminants" (2020). Articles. 89.

https://arrow.tudublin.ie/ittsciart/89

This Article is brought to you for free and open access by the School of Science and Computing at ARROW@TU Dublin. It has been accepted for inclusion in Articles by an authorized administrator of ARROW@TU Dublin. For more information, please contact arrow.admin@tudublin.ie, aisling.coyne@tudublin.ie, gerard.connolly@tudublin.ie.

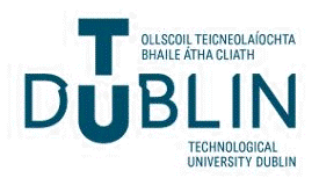


DOI 10.1007/s10986-019-09455-8

Lithuanian Mathematical Journal, Vol. 60, No. 1, January, 2020, pp. 1-8

\title{
New estimates for the number of integer polynomials with given discriminants
}

\author{
Natalia Budarina $^{\mathrm{a}}$, Vasilii Bernik ${ }^{\mathrm{b}}$, and Hugh O'Donnell ${ }^{\mathrm{c}}$ \\ ${ }^{a}$ Dundalk Institute of Technology, Dublin Road, Dundalk, Co. Louth, Republic of Ireland \\ ${ }^{\mathrm{b}}$ Institute of Mathematics, Surganova str. 11, 220072, Minsk, Belarus \\ ${ }^{c}$ Dublin Institute of Technology, Dublin, D2, Republic of Ireland \\ (e-mail: buda77@mail.ru; bernik.vasili@mail.ru; hugh.odonnell@dit.ie)
}

Received September 25, 2018; revised August 4, 2019

\begin{abstract}
In this paper, we propose a new method of upper bounds for the number of integer polynomials of the fourth degree with a given discriminant. By direct calculation similar results were established by H. Davenport and D. Kaliada for polynomials of second and third degrees.
\end{abstract}

MSC: $11 \mathrm{~J} 83,11 \mathrm{~J} 68$

Keywords: Diophantine approximation, discriminant of polynomials

\section{Introduction}

Denote by $\mathcal{P}_{n}$ the class of integer polynomials $P$ of degree $n$. In what follows, we use the Vinogradov symbols $\ll($ and $\gg)$ where $a \ll b$ means that there exists a constant $C$ such that $a \leqslant C b$. If $a \ll b \ll a$, then we write $a \asymp b$. We denote the cardinality of a set $B$ by \#B. Positive constants that depend only on $n$ will be denoted by $c(n)$; where necessary, these constants will be numbered $c_{j}(n), j=1,2, \ldots$.

The discriminant of a polynomial $P(x)=a_{n} x^{n}+a_{n-1} x^{n-1}+\cdots+a_{1} x+a_{0} \in \mathcal{P}_{n}$ is defined by

$$
D(P)=a_{n}^{2 n-2} \prod_{1 \leqslant i<j \leqslant n}\left(\alpha_{i}-\alpha_{j}\right)^{2}
$$

where $\alpha_{1}, \alpha_{2}, \ldots, \alpha_{n} \in \mathbb{C}$ are the roots of $P$. Let $H(P)=\max _{0 \leqslant j \leqslant n}\left|a_{j}\right|$ denote the standard (naive) height of $P=\sum_{i=0}^{n} a_{i} x^{i}$. Given a parameter $Q \in \mathbb{N}_{>1}$, let

$$
\mathcal{P}_{n}(Q)=\left\{P(x) \in \mathcal{P}_{n}: H(P) \leqslant Q\right\}
$$

denote the set of integer polynomials $P$ of degree $n$ and height $H(P) \leqslant Q$. If $P$ has no repeated roots, then $D(P) \neq 0$. It is well known [16] that $D(P)$ can be represented as a determinant of order $2 n-1$, which consists 
of the coefficients of $P$. Hence, whenever $D(P) \neq 0$, we have that $|D(P)| \geqslant 1$ and $|D(P)|$ is bounded from above in terms of the height and degree of the polynomial $P$. We easily verify that for every $n \geqslant 2$, there exists a constant $c_{1}>0$ that depends on $n$ only such that for any $P \in \mathcal{P}_{n}(Q)$, we have that

$$
1 \leqslant|D(P)|<c_{1} Q^{2 n-2}
$$

The properties and estimates for $D(P)$ imply the estimates for $\left|x-\alpha_{1}\right|$, where $x \in \mathbb{R}$, and $\alpha_{1}$ is the root of $P$ closest to $x$ (see $[9,10,15]$ ). These estimates were crucial to prove Mahler's conjecture in the case $n=2,3$. In a more systematic way, the relation between $\left|x-\alpha_{1}\right|$ and $D(P)$ was investigated by Sprindzuk [15] and others $[2,3,4,5,6,11,12,13,14]$. In recent years, the problem of counting polynomials with a small discriminant $D(P)$ has become a new branch of the theory of Diophantine approximations.

Given $v \in \mathbb{R}_{\geqslant 0}$, define the subset of $\mathcal{P}_{n}(Q)$ as follows:

$$
\mathcal{P}_{n}(Q, v)=\left\{P(x) \in \mathcal{P}_{n}(Q): 1 \leqslant|D(P)|<Q^{2 n-2-2 v}\right\}
$$

Establishing the correct lower and upper bounds for $\# \mathcal{P}_{n}(Q, v)$ is the goal of this branch of Diophantine approximations. We now briefly recall the results obtained to date. In the case of quadratic polynomials, it was shown in [13] that

$$
\# \mathcal{P}_{2}(Q, v) \asymp Q^{3-2 v}, \quad 0<v<\frac{3}{4} .
$$

In the case of cubic polynomials, it was established in [14] that

$$
\# \mathcal{P}_{3}(Q, v) \asymp Q^{4-5 v / 3}, \quad 0 \leqslant v<\frac{3}{5} .
$$

Establishing the correct lower bounds for arbitrary $n$ has been the subject of numerous papers including [2,3, $6,13,14]$. The most general and best estimate was found in [3], where it was shown that

$$
\# \mathcal{P}_{n}(Q, v)>c_{2} Q^{n+1-(n+2) v / n}, \quad 0 \leqslant v \leqslant n-1
$$

The lower bound (1.2) for the full range of $v, 0 \leqslant v \leqslant n-1$, was obtained for the polynomials that have all $\alpha_{2}, \ldots, \alpha_{n}$ roots close to $\alpha_{1}$ and $x$. The method for constructing a large number of polynomials $P \in \mathcal{P}_{n}(Q, v)$ is based on the results from [1]. Moreover, the following two propositions are key elements of the method for obtaining the lower bound (1.2).

Proposition 1. (See [3].) Let $n \geqslant 2$, and let $v_{0}, v_{1}, \ldots, v_{n}$ be a collection of real numbers such that

$$
v_{0}+v_{1}+\cdots+v_{n}=0 \text { and } v_{0} \geqslant v_{1} \geqslant \cdots \geqslant v_{n} \geqslant-1 .
$$

Then there are positive constants $c_{3}$ and $c_{4}$ depending on $n$ only with the following property. For any interval $J \subset[1 / 2,1 / 2]$, there is a sufficiently large $Q_{0}$ such that for all $Q>Q_{0}$, there is a measurable set $G_{J} \subset J$ satisfying $\left|G_{J}\right| \geqslant|J| / 2$ such that for every $x \in G_{J}$, there are $n+1$ linearly independent primitive irreducible polynomials $P \in \mathbb{Z}[x]$ of degree exactly $n$ such that

$$
c_{3} Q^{-v_{0}} \leqslant|P(x)| \leqslant c_{4} Q^{-v_{0}}, \quad c_{3} Q^{-v_{j}} \leqslant\left|P^{(j)}(x)\right| \leqslant c_{4} Q^{-v_{j}}, \quad 1 \leqslant j \leqslant n .
$$


Proposition 2. (See [3].) Let $n$ and $v_{j}$ be as in Proposition 1. Let

$$
d_{j}=v_{j-1}-v_{j}, \quad 1 \leqslant j \leqslant n .
$$

Suppose that $d_{1} \geqslant d_{2} \geqslant \cdots \geqslant d_{n} \geqslant 0$ and that for some $x \in \mathbb{C}$ and $Q>1$, inequalities (1.3) are satisfied by some polynomial $P$ over $\mathbb{C}$ of degree $\operatorname{deg} P=n$. Then there are roots $\alpha_{1}, \ldots, \alpha_{n} \in \mathbb{C}$ of $P$ such that

$$
\left|x-\alpha_{j}\right| \leqslant c_{5, j} Q^{-d_{j}}, \quad 1 \leqslant j \leqslant n
$$

where

$$
\begin{aligned}
c_{5,1} & =n c_{4} c_{3}^{-1}, \\
c_{5, j+1} & =\max \left(\frac{2 c_{4} n !}{c_{3}(j+1) !(n-j-1) !}, \frac{2 c_{5, j} n !}{j !(n-j !)}\right), \quad 1 \leqslant j \leqslant n-1 .
\end{aligned}
$$

It is much harder to get upper bounds for $\# \mathcal{P}_{n}(Q, v)$ with arbitrary $n$. Note that the range of $v$ depends on the number of roots of the polynomial close to $\alpha_{1}$. For example, if only one root $\alpha_{2}$ is close to $\alpha_{1}$, then the range for $v$ is $0 \leqslant v \leqslant n / 2$.

For results in the $p$-adic case, see [7]. The upper and lower bounds for the number of polynomials having small discriminants in terms of the Euclidean and $p$-adic metrics simultaneously are obtained in $[5,11]$.

Let $\alpha_{1}, \ldots, \alpha_{n}$ be the roots of the polynomial $P \in \mathcal{P}_{n}$. An upper bound for the number of integer cubic polynomials with a given discriminant is obtained in [4], where it is established that

$$
\# \mathcal{P}_{3}^{\prime}(Q, v) \ll Q^{4-5 v / 3+\epsilon}, \quad 0 \leqslant v \leqslant 2, \forall \epsilon>0
$$

where $\mathcal{P}_{3}^{\prime}(Q, v)$ is a subclass of $\mathcal{P}_{3}(Q, v)$ with a special distribution of roots. The first step of the proof is the ordering the roots $\alpha_{1}, \alpha_{2}, \alpha_{3}$ with respect to one of them $\alpha_{j}$, which will denote by $\alpha_{1}$, in such way that

$$
\left|\alpha_{1}-\alpha_{2}\right| \leqslant\left|\alpha_{1}-\alpha_{3}\right|, \quad\left|\alpha_{1}-\alpha_{3}\right| \asymp\left|\alpha_{2}-\alpha_{3}\right| .
$$

In the case of the polynomials of fourth degree, we will have another principal case for the ordering of the roots:

$$
\begin{aligned}
& \left|\alpha_{1}-\alpha_{2}\right| \leqslant\left|\alpha_{1}-\alpha_{3}\right| \leqslant\left|\alpha_{1}-\alpha_{4}\right|, \\
& \left|\alpha_{1}-\alpha_{2}\right| \ll\left|\alpha_{3}-\alpha_{4}\right| \ll\left|\alpha_{2}-\alpha_{3}\right| \ll\left|\alpha_{1}-\alpha_{3}\right| .
\end{aligned}
$$

Other cases are similar to (1.4).

Let $\alpha_{1 j}, \ldots, \alpha_{n j}$ be the roots of the polynomial $P_{j} \in \mathcal{P}_{n}$ ordered according to (1.4) or (1.5) depending on the degree of $P_{j}$. For $n=3$, the polynomials $P_{j}$ are expanded into Taylor series in a neighbourhood of $\alpha_{1 j}$, and the absolute values of $P_{j}$ are estimated from above. Then we form the new polynomials $R_{j+1}=P_{j+1}-P_{j}$ of degree $\operatorname{deg} R_{j+1}<n$ from the polynomials $P_{j}$ with the same oldest coefficients.

For the polynomials of fourth degree, in case (1.4), from the estimates $\left|P_{j}\right|$ in a neighbourhood of $\alpha_{1 j}$ we cannot get strong estimates for $\left|P_{j}\right|$ in a neighbourhood of $\alpha_{3 j}$. Therefore the expansion into Taylor series must be carried out in a neighbourhood of $\alpha_{1 j}$ and in a neighbourhood of $\alpha_{3 j}$.

The partition of the roots $\alpha_{j}$ into the clusters is possible for $n=5,6$, but for the arbitrary $n$, we did not find a convenient method to classify the roots. Therefore, from now on, $n=4$ and the roots $\alpha_{j}$ satisfy (1.5). Let $\mathcal{P}_{4}^{\prime}(Q, v)$ denote the set of polynomials $P \in \mathcal{P}_{4}(Q, v)$ with distinct roots satisfying (1.5). In this paper, we obtain an upper bound for the number of polynomials $P \in \mathcal{P}_{4}^{\prime}(Q, v)$.

Theorem 1. For any $\epsilon>0$ and any sufficiently large $Q$, we have the estimate

$$
\# \mathcal{P}_{4}^{\prime}(Q, v)<Q^{5-3 v / 2+\epsilon}, \quad 0 \leqslant v \leqslant 1
$$




\section{Auxiliary statements}

Let $P \in \mathcal{P}_{4}^{\prime}(Q, v)$ have complex distinct roots $\alpha_{1}, \alpha_{2}, \alpha_{3}, \alpha_{4}$. Let

$$
\left|\alpha_{1}-\alpha_{i}\right|=Q^{-\rho_{i}}, \quad 2 \leqslant i \leqslant 4, \rho_{2} \geqslant \rho_{3} \geqslant \rho_{4},
$$

and

$$
\left|\alpha_{3}-\alpha_{4}\right|=Q^{-\rho_{5}}
$$

Similar to other problems of the metric theory regarding polynomials, we assume that $\left|a_{4}(P)\right| \gg H(P)$. If the polynomial $P$ does not satisfy the last condition, then the transformation $S(x)=P(x+m)$ for some $0 \leqslant m \leqslant 4$ can be performed followed by an inversion to obtain $U(x)=x^{4} S(1 / x)$. Therefore this new polynomial $U(x)=\sum_{j=0}^{4} b_{j} x^{j}$ satisfies $\left|b_{4}\right| \gg H(S) \asymp H(P)$. For more details, see [15]. If $P$ satisfies $\left|a_{4}(P)\right| \gg H(P)$, then $\left|\alpha_{i}\right| \leqslant c_{6}, 1 \leqslant i \leqslant 4$, and $\left|\alpha_{i}-\alpha_{j}\right| \leqslant 2 c_{6}$ for $1 \leqslant i<j \leqslant 4$. Therefore $\rho_{i} \geqslant \epsilon_{1}$, $2 \leqslant i \leqslant 5$, for any $\epsilon_{1}>0$ and any sufficiently large $Q$.

For a given number $\epsilon_{1}>0$, let $T=\left[\epsilon_{1}^{-1}\right]+1$, where $[a]$ is the integer part of $a \in \mathbb{R}$. For a polynomial $P \in \mathcal{P}_{4}(Q, v)$, the real numbers $\rho_{i}, i=2,3,4,5$, were defined in (2.1). Also define the integers $l_{i}$ by

$$
\frac{l_{i}-1}{T}<\rho_{i} \leqslant \frac{l_{i}}{T}, \quad i=2,3,4,5 .
$$

It is not difficult to show that the number of vectors $\bar{l}=\left(l_{2}, l_{3}, l_{4}, l_{5}\right)$ is finite, depends only on $\epsilon_{1}$, and does not depend on $Q$ and $H(P)$.

In order for the polynomial $P(x)$ to belong to the class $\mathcal{P}_{4}^{\prime}(Q, v)$, it is necessary and sufficient that the inequality

$$
\rho_{2}+2 \rho_{3}+2 \rho_{4}+\rho_{5} \geqslant v
$$

holds. Note that inequality (2.3) follows from (1.1), (1.5), (2.1), (2.2), and the triangle inequalities for the roots of the polynomial $P$. For (2.3), the inequality

$$
\frac{l_{2}}{T}+\frac{2 l_{3}}{T}+\frac{2 l_{4}}{T}+\frac{l_{5}}{T} \geqslant v+6 \epsilon_{1}
$$

is sufficient. By (1.1), (2.1), and (2.2) we have

$$
\rho_{2}+2 \rho_{3}+2 \rho_{4}+\rho_{5} \leqslant 3
$$

For the roots of $P \in \mathcal{P}_{4}$, we define the sets

$$
S\left(\alpha_{j}\right)=\left\{x \in \mathbb{R}:\left|x-\alpha_{j}\right|=\min _{1 \leqslant i \leqslant 4}\left|x-\alpha_{i}\right|\right\}, \quad 1 \leqslant j \leqslant 4 .
$$

Lemma 1. Let $\alpha_{1}$ be a complex root of an integer polynomial $P \in \mathcal{P}_{4}$, and let $x \in S\left(\alpha_{1}\right)$. Then

$$
\left|x-\alpha_{1}\right| \leqslant \min _{2 \leqslant j \leqslant 4}\left(2^{4-j}|P(x)|\left|P^{\prime}\left(\alpha_{1}\right)\right|^{-1} \prod_{k=2}^{j}\left|\alpha_{1}-\alpha_{k}\right|\right)^{1 / j}
$$

for $P^{\prime}\left(\alpha_{1}\right) \neq 0$.

Lemma 1 is proved in [10]. 
Lemma 2. Fix $\delta>0$ and $Q>Q_{0}(\delta)$. Suppose that the polynomials $P(x), T(x) \in \mathcal{P}_{k}(Q), k \leqslant 4$, have the same vector $\bar{l}$ and have no common roots. Let I denote interval of length $|I|=Q^{-\gamma}$ with $\gamma \in \mathbb{R}_{+}$. If there exists a real number $\tau>0$ such that for all $x \in I$,

$$
\max _{x \in I}(|P(x)|,|T(x)|)<Q^{-\tau},
$$

then

$$
\tau+1+2 \sum_{j=1}^{k} \max (\tau+1-j \gamma, 0)<2 k+\delta .
$$

Lemma 2 can be proved similarly to Lemma 3 in [5]. In this case, we need to add the summands related to the root $\alpha_{4}$.

To prove Theorem 1, we need to consider a generalization of Lemma 2 for the simultaneous approximations of the polynomials on two intervals (see Lemma 3). We consider a new classification of the roots $\alpha_{i}, 1 \leqslant i \leqslant 4$, of $P \in \mathcal{P}_{4}^{\prime}(Q)$ with respect to $\alpha_{1}$ (as before) and $\alpha_{3}$ simultaneously. We obtain

$$
\begin{aligned}
& \left|\alpha_{1}-\alpha_{2}\right| \leqslant\left|\alpha_{1}-\alpha_{3}\right| \leqslant\left|\alpha_{1}-\alpha_{4}\right|, \\
& \left|\alpha_{3}-\alpha_{4}\right| \leqslant\left|\alpha_{3}-\alpha_{2}\right| \leqslant\left|\alpha_{3}-\alpha_{1}\right| .
\end{aligned}
$$

Let $\left|\alpha_{3}-\alpha_{2}\right|=Q^{-\rho_{6}}$ and define the integer $l_{6}$ by $\left(l_{6}-1\right) / T<\rho_{6} \leqslant l_{6} / T$. It is not difficult to see that by $(1.5)$

$$
\rho_{4} \leqslant \rho_{3} \leqslant \rho_{2}, \quad \rho_{3} \leqslant \rho_{6} \leqslant \rho_{5},
$$

where $\rho_{i}, 2 \leqslant i \leqslant 5$, are defined in (2.1)-(2.2). We also define the vector $\bar{l}^{\prime}=\left(\bar{l}, l_{6}\right)$. Define the class $\mathcal{P}_{4, \bar{l}^{\prime}}^{\prime}(Q, v)$ consisting of the polynomials $P \in \mathcal{P}_{4}^{\prime}(Q, v)$ corresponding to a vector $\bar{l}^{\prime}$.

Lemma 3. Fix $\delta>0$ and $Q>Q_{0}(\delta)$. Suppose that the polynomials $P(t), T(t) \in \mathcal{P}_{k}(Q), k \leqslant 4$, have the same vector $\bar{l}^{\prime}$ and have no common roots in the rectangle $I_{1} \times I_{2}$, where $\left|I_{1}\right|=Q^{-\gamma_{1}}$ and $\left|I_{2}\right|=Q^{-\gamma_{2}}$ with $\gamma_{j} \in \mathbb{R}_{+}, j=1,2$. Furthermore, let $P(t)$ and $T(t)$ satisfy the system of inequalities

$$
\max _{x \in I_{1}}(|P(x)|,|T(x)|)<Q^{-\tau_{1}}, \quad \max _{y \in I_{2}}(|P(y)|,|T(y)|)<Q^{-\tau_{2}} .
$$

Then for any $\delta>0$ and $Q>Q_{0}(\delta)$, we have the inequality:

$$
\tau_{1}+\tau_{2}+2+l_{2}+2 l_{3}+3 l_{4}+l_{5}<2 k+\delta .
$$

The proof of Lemma 3 follows from the new classification (2.5) of the roots of polynomials, using inequalities (2.6) and (2.7), and can be proved similarly to Lemma 2 in [8].

\section{Proof of Theorem 1}

Assume that estimate (1.6) does not hold, so that

$$
\# \mathcal{P}_{4}^{\prime}(Q, v) \geqslant Q^{5-3 v / 2+\epsilon} .
$$

Consider two intervals $I_{1}, I_{2} \subset \mathbb{R}$ with $\left|I_{1}\right|=Q^{-l_{2} / T}$ and $\left|I_{2}\right|=Q^{-l_{5} / T}$. We will say that the polynomial $P$ belongs to $M=I_{1} \times I_{2}$ if $\left(\alpha_{1}, \alpha_{3}\right) \in M$, where $\alpha_{1}$ and $\alpha_{3}$ are the roots of $P$ in the ordering (1.5). From (3.1) it follows that there exist rectangles $I_{1} \times I_{2}$ that contain at least

$$
\Delta=Q^{5-3 v / 2-l_{2} / T-l_{5} / T+\epsilon}
$$


polynomials $P \in \mathcal{P}_{4}^{\prime}(Q, v)$ satisfying (2.7). Fix one of these rectangles, say $M$. Since $\# \bar{l}^{\prime} \ll 1$, there exists a vector $\bar{l}^{\prime}$ satisfying $(2.3)$ such that

$$
\# \mathcal{P}_{4, \bar{l}^{\prime}}^{\prime}(Q, v, M) \gg Q^{5-3 v / 2+\epsilon-l_{2} / T-l_{5} / T+\epsilon},
$$

where $\mathcal{P}_{4, \bar{l}^{\prime}}^{\prime}(Q, v, M)$ denotes the subset of $\mathcal{P}_{4, \bar{l}^{\prime}}^{\prime}(Q, v)$ consisting of polynomials $P$ belonging to $M$. Fix the vector $\bar{l}^{\prime}$ and set

$$
h=5-\frac{3 v}{2}-\frac{l_{2}}{T}-\frac{l_{5}}{T}+\frac{\epsilon}{2}
$$

By (2.4) we have

$$
\frac{l_{2}}{T}+\frac{2 l_{3}}{T}+\frac{2 l_{4}}{T}+\frac{l_{5}}{T} \leqslant 3 .
$$

From (3.2) we obtain that $h>0$ for $v \leqslant 4 / 3$.

Expand the polynomial $P \in \mathcal{P}_{4, \bar{l}^{\prime}}^{\prime}(Q, v, M)$ into its Taylor series in a neighbourhood of $\alpha_{1}$ to obtain

$$
\begin{aligned}
P(x)= & P\left(\alpha_{1}\right)+P^{\prime}\left(\alpha_{1}\right)\left(x-\alpha_{1}\right) \\
& +\frac{1}{2} P^{\prime \prime}\left(\alpha_{1}\right)\left(x-\alpha_{1}\right)^{2}+\frac{1}{6} P^{\prime \prime \prime}\left(\alpha_{1}\right)\left(x-\alpha_{1}\right)^{3}+\frac{1}{24} P^{(4)}\left(\alpha_{1}\right)\left(x-\alpha_{1}\right)^{4}
\end{aligned}
$$

Estimating each term gives

$$
\begin{aligned}
\left|P^{\prime}\left(\alpha_{1}\right)\left(x-\alpha_{1}\right)\right| \leqslant & \left|a_{4}\right| \cdot\left|\alpha_{1}-\alpha_{2}\right| \cdot\left|\alpha_{1}-\alpha_{3}\right| \cdot\left|\alpha_{1}-\alpha_{4}\right| \cdot\left|x-\alpha_{1}\right| \\
\leqslant & Q^{1-\rho_{2}-\rho_{3}-\rho_{4}-l_{2} / T}<Q^{1-2 l_{2} / T-l_{3} / T-l_{4} / T+3 \epsilon_{1}}, \\
\left|P^{\prime \prime}\left(\alpha_{1}\right)\left(x-\alpha_{1}\right)^{2}\right| \leqslant & 6\left|a_{4}\right| \max \left(\left|\alpha_{1}-\alpha_{2}\right|\left|\alpha_{1}-\alpha_{3}\right|,\left|\alpha_{1}-\alpha_{2}\right|\left|\alpha_{1}-\alpha_{4}\right|,\left|\alpha_{1}-\alpha_{3}\right|\left|\alpha_{1}-\alpha_{4}\right|\right) \\
& \times\left|x-\alpha_{1}\right|^{2} \\
< & 6 Q^{1-2 l_{2} / T-l_{3} / T-l_{4} / T+2 \epsilon_{1}}, \\
\left|P^{\prime \prime \prime}\left(\alpha_{1}\right)\left(x-\alpha_{1}\right)^{3}\right| \leqslant & 18\left|a_{4}\right| \max \left(\left|\alpha_{1}-\alpha_{2}\right|,\left|\alpha_{1}-\alpha_{3}\right|,\left|\alpha_{1}-\alpha_{4}\right|\right) \cdot\left|x-\alpha_{1}\right|^{3} \\
< & 18 Q^{1-3 l_{2} / T-l_{4} / T+\epsilon_{1}} \\
\left|P^{(4)}\left(\alpha_{1}\right)\left(x-\alpha_{1}\right)^{4}\right| \leqslant & 24\left|a_{4}\right|\left|x-\alpha_{1}\right|^{4} \leqslant 24 Q^{1-4 l_{2} / T}
\end{aligned}
$$

for $x \in I_{1}$. Thus

$$
|P(x)| \ll Q^{1-2 l_{2} / T-l_{3} / T-l_{4} / T+3 \epsilon_{1}}, \quad x \in I_{1} .
$$

Also develop the polynomial $P$ as Taylor series on the interval $I_{2}$ at the point $\alpha_{3}$ and obtain the upper bounds for all terms in the series. Thus we obtain

$$
|P(y)| \ll Q^{1-2 l_{5} / T-l_{3} / T-l_{6} / T+3 \epsilon_{1}}, \quad y \in I_{2}
$$

Further, for $Q^{h}$ polynomials $P$, we use the Dirichlet box principle. We will assume that the fractional part of $h$ does not exceed $\epsilon_{1}$. If the last condition is not satisfied, then we rewrite $h$ as $h=[h]+\{h\}$. As a result, using the number $Q^{[h]}$, we reduce the degree of polynomials, and using the number $Q^{\{h\}}$, we reduce the height 
of polynomials $R_{j+1}(t)=P_{j+1}(t)-P_{1}(t), j=1,2, \ldots$, as in [5]. Therefore the new polynomials $R_{j}$ satisfy

$$
\begin{gathered}
\left|R_{j}(x)\right| \ll Q^{1-2 l_{2} / T-l_{3} / T-l_{4} / T+3 \epsilon_{1}}, \quad x \in I_{1}, \\
\left|R_{j}(y)\right| \ll Q^{1-2 l_{5} / T-l_{3} / T-l_{6} / T+3 \epsilon_{1}}, \quad y \in I_{2}, \\
H\left(R_{j}\right) \leqslant Q^{1-\epsilon_{1}}, \quad \operatorname{deg} R_{j} \leqslant 4-\left(5-\frac{3 v}{2}-\frac{l_{2}}{T}-\frac{l_{5}}{T}+\frac{\epsilon}{2}-\epsilon_{1}\right) .
\end{gathered}
$$

If there exist two polynomials $R_{1}$ and $R_{2}$ with no common roots, then Lemma 3 can be applied. The values of $\tau_{1}$ and $\tau_{2}$ are found from estimates (3.3) and (3.4). Thus

$$
\tau_{1}=\frac{-1+2 l_{2} / T+l_{3} / T+l_{4} / T-3 \epsilon_{1}}{1-\epsilon_{1}} \quad \text { and } \quad \tau_{2}=\frac{-1+2 l_{5} / T+l_{3} / T+l_{6} / T-3 \epsilon_{1}}{1-\epsilon_{1}} .
$$

The left-hand side of (2.8) is equal to

$$
\frac{3 l_{2} / T+4 l_{3} / T+4 l_{4} / T+3 l_{5} / T+l_{6} / T-6 \epsilon_{1}}{1-\epsilon_{1}} .
$$

This leads to a contradiction in (2.8) for $v \leqslant 1$ and $\delta \leqslant \epsilon-2 \epsilon_{1}$.

If, among polynomials $R_{j}(t)$, there exist no two polynomials without common roots, then the polynomials $R_{j}(t)$ are reducible. It is not difficult to see that $\operatorname{deg} R_{j} \leqslant 2$ for $v \leqslant 1$. Thus the polynomials $R_{j}(t)$ are decomposed into the product of two linear polynomials. Again, as, for example, in [4], we will use Lemma 2 to get a contradiction.

\section{References}

1. V. Beresnevich, Rational points near manifolds and metric Diophantine approximation, Ann. Math. (2), 175(1): $187-235,2012$.

2. V. Beresnevich, V. Bernik, and F. Götze, Simultaneous approximations of zero by an integral polynomial, its derivative, and small values of discriminants, Dokl. Nats. Akad. Nauk Belarusi, 54(2):26-28, 2010.

3. V. Beresnevich, V. Bernik, and F. Götze, Integral polynomials with small discriminants and resultants, Adv. Math., 298:393-412, 2016.

4. V. Bernik, N. Budarina, and F. Götze, Exact upper bounds for the number of the polynomials with given discriminant, Lith. Math. J., 57(3):283-293, 2017.

5. V. Bernik, N. Budarina, and H. O'Donnell, Discriminants of polynomials in the Archimedean and non-Archimedean metrics, Acta Math. Hung., 154(2):265-278, 2018.

6. V. Bernik, F. Götze, and O. Kukso, Lower bounds for the number of integral polynomials with given order of discriminants, Acta Arith., 133:375-390, 2008.

7. V. Bernik, F. Götze, and O. Kukso, On the divisibility of the discriminant of an integral polynomial by prime powers, Lith. Math. J., 48(4):380-396, 2008.

8. V. Bernik and S. Mc Guire, How small can polynomials be in an interval of given length?, Glasg. Math. J., 2019, available from: https://doi.org/10.1017/S0017089519000077.

9. V.I. Bernik, An application of Hausdorff dimension in the theory of Diophantine approximation, Acta Arith., 42:219253, 1983 (in Russian).

10. V.I. Bernik, The exact order of approximating zero by values of integral polynomials, Acta Arith., 53:17-28, 1989 (in Russian). 
11. N. Budarina, D. Dickinson, and Y. Jin, On the number of polynomials with small discriminants in the Euclidean and p-adic metrics, Acta Math. Sin., Engl. Ser., 28(3):469-476, 2012.

12. Y. Bugeaud, Approximation by Algebraic Numbers, Camb. Tracts Math., Vol. 160, Cambridge Univ. Press, Cambridge, 2004.

13. F. Götze, D. Kaliada, and M. Korolev, On the number of integral quadratic polynomials with bounded heights and discriminants, 2013, arXiv:1308.2091.

14. F. Götze, D. Kaliada, and O. Kukso, The asymptotic number of integral cubic polynomials with bounded heights and discriminants, Lith. Math. J., 54(2):150-165, 2014.

15. V.G. Sprindzuk, Mahler's Problem in Metric Number Theory, Transl. Math. Monogr., Vol. 25, AMS, Providence, RI, 1969. Translated from the Russian by B. Volkmann.

16. B.L. Van Der Waerden, Algebra, Springer, Berlin, Heidelberg, 1971. 